\title{
Congenital rubella syndrome exposure in a pediatric hospital: experience from developing world
}

\author{
D Sureshkumar*, R Gopalakrishnan, L Jessani \\ From 3rd International Conference on Prevention and Infection Control (ICPIC 2015) \\ Geneva, Switzerland. 16-19 June 2015
}

\section{Introduction}

Congenital rubella syndrome (CRS) is a rare disorder with devastating ocular and systemic consequences. Although efforts to eradicate the disease have been in place for some time, developing world continue to be affected by this disease

\section{Objectives}

We describe an exposure to a six month old infant with congenital rubella syndrome (CRS) in the pediatric hospital and the successful management of exposure and prevention of rubella cases among exposed staff with limited resources.

\section{Methods}

In January 2015, a six month old infant with a patent ductus arteriosus was admitted to a 50 bedded tertiary referral pediatric hospital in South India. The infant was seen \& managed by cardiologist initially followed by cardio thoracic surgery team, pediatricians, intensivists and ophthalmologist. Although CRS was mentioned in the differential diagnoses of several physicians, the patient was not isolated until the 8th day of hospitalization. This may be part due to the unrecognized nature \& duration of infectivity of CRS.

\section{Results}

Totally thirty five health care workers (HCWs) exposed to CRS child during his initial 8 days of stay in the hospital. Majority of the exposure happened during his stay in multi bedded general ward (18/35), followed by cardio thoracic intensive care unit (4/35) and in the operating room (3/35). Two of the exposed employees were pregnant that time. An attempt was made to prevent an outbreak of additional exposures by the isolation (both contact \& droplet precautions) of the infant, rubella Ig $\mathrm{G}$ antibody testing of both pregnant health care workers with unknown or uncertain history of rubella vaccination (both were immune) and prompt administration of rubella vaccine to significantly exposed persons.

\section{Conclusion}

Outbreaks of CRS that occur in pediatric hospitals in the developing world are of special concern. Testing of all employees for rubella antibody and immunization of those determined to be seronegative should be considered. Infants with congenital rubella syndrome shed rubella virus in large quantity for prolonged periods in urine and saliva. They need to be kept in both contact \& droplet isolation for minimum 1 year to avoid unprotected exposures among HCWs

\section{Disclosure of interest \\ None declared.}

Published: 16 June 2015

doi:10.1186/2047-2994-4-S1-P260

Cite this article as: Sureshkumar et al:: Congenital rubella syndrome exposure in a pediatric hospital: experience from developing world. Antimicrobial Resistance and Infection Control 2015 4(Suppl 1):P260.

Infectious Disease, Apollo Hospitals, Chennai, India 